\title{
POWER ELECTRONICS: HISTORICAL NOTES, RECENT ADVANCES AND CONTEMPORARY CHALLENGES
}

\author{
Don Tan \\ e-mail: dong.tan@ieee.org
}

\begin{abstract}
This paper attempts to summarize 10 major contemporary challenges for power electronics and system technology (PEAS technology). Historical background is first reviewed together with most recent technological advances. Recent advances are then discussed in detail. A recent breakthrough in megawatt system design and design verification is highlighted. We then articulate the challenges that PEAS technology is facing contemporarily. They are: Powering smart buildings, smart factories, and smart infrastructure, Renewable energy integration and structured microgrids, EV drives and vehicle power systems, Ultra-fast and ultra-efficient chargers, Appliances - "White goods," Server and data center power systems' Wireless power transfer, Powering IoT and wireless sensor networks, Storage and "power dump," and ATGC (All things grid connected), system integration, and dynamic control. These 10 areas present technical barriers for us to overcome and anticipated progress will help define the future of power electronics and its impact to the power and energy industry at large.
\end{abstract}

Keywords - Electronic power grid (eGrid), Flexible autonomous power substations (FAPS), Flexible electronic large power transformers (FeLPTs), Power electronics, Power electronics and systems (PEAS), Power supply on chip (PwrSoc), Wireless power.

\section{INTRODUCTION}

Power electronics is experiencing phenomenal growth in all aspects of the technology: from controller ICs to $\mathrm{Si}$ devices to WBG devices, from low-power micro-modules to medium-power bricks to high-power (megawatt) modular multilevel switching cells; from smart phones to smart buildings to smart cities; from smart inverters to flexible electronics large transformers to flexible power substations; from smart drives to drive/machine integration to wireless power transfer to electric cars; and to transportation electrification.

Among this dazzling technology advancement and changes, it is crucially important for a research or a technologist to be able to see the underlying technological trends and future directions. Only when equipped with these, one can anticipate what around the corners for the big technological changes happening to all of us. Recent attempts by the same author(s) provided pretty extensive

Invited paper; accepted for publication $12 / 18 / 2020$, by recommendation of
Editor Demercil de Souza Oliveira Jr. http://dx.doi.org/10.18618/REP.2020.4.00701 discussion on the topics [1-6]. This article presents an update and an extension to the cotemporary trends and challenges.

The discussion is organized into three (3) major topics for detailed discussion. After a brief introduction in Section 1, we begin with historical notes in Section 2, where we show the power electronic can be traced back as early as 1903 . We then focus on recent advances Section 3, particularly in WBG device, multilevel conversion technique, a recent breakthrough in megawatt power design verification and PwrSoc technology. We then discuss In Section 4 the top ten contemporary technological trends. They are: Powering smart buildings, smart factories, and smart infrastructure, Renewable energy integration and structured microgrids, EV drives and vehicle power systems, Ultra-fast and ultraefficient chargers, Appliances - "White goods," Server and data center power systems' Wireless power transfer, Powering IoT and wireless sensor networks, Storage and "power dump," and ATGC (All things grid connected), system integration, and dynamic control. We present a summary in Section 5 and reference section for relevant papers, reports, and Internet items.

\section{HISTORIC NOTES}

We begin with some historical notes to lay some background for today's technological trends in power electronics and systems (PEAS) technologies.

Power electronics (PE) was established formally as technical field within IEEE in 1986 by the establishment of IEEE Technical Council on Power Electronics. But its roots can be traced back to early days of electrical engineering. The mercury valve was invented by P. C. Hewitt in 1902 [7]. This is the primary technology for high power rectification. Early prototypes are shown in Figure 1. The thyratron extended the concept of mercury arc valve with fast switching (no amplification) and compact in size [8].
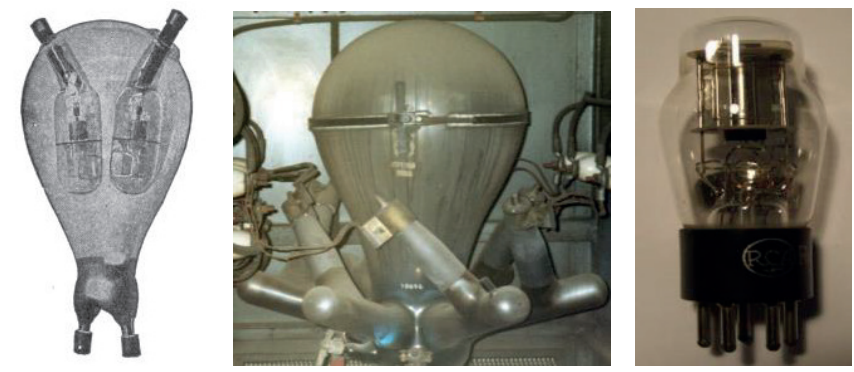

Fig. 1. Mercury Arc Valve (Rectifier): The original prototype by P. C. Hewitt in 1902 (left) and an old industry product (center) and a more recent RC85 thyratron $[7,8]$. 
The Greinacher voltage multiplier was introduced in 1914 [9], where two nonlinear impedances, labeled $\mathrm{Z}$ respectively, were used to step up the voltage level, as shown in Figure 2.

In English literature, the circuit was later known as the Cockcroft-Walton Multiplier, because of his Nobel Prize winning article in 1932 [10]. The circuit diagram and hardware in the lab are shown in Figure 3

The 1940's saw two major switching elements used: The magnetic amplifier (MagAmp) and the vacuum tube. Refer to Figure 4. The magnetic amplifier is an electromagnetic device controlled by a dc bias current. Because of its high reliability, it was widely used for application where long life and high reliability are required [11]. Figure 5 shows a line of various vacuum tubes [12]. The vacuum tube was the workhorse from the 1940's to the 1960's for radio frequency power amplification.

The silicon-controlled rectifier (SCR) was introduced in the 1950's. An IEEE technical milestone was formally established in 2019 by the Power Electronics Society and the IEEE Board of Director at the GE site where the first SCR were manufactured [13]. The Silicon bipolar transistor was introduced in the 1960's. The RCA 2N3055 was the first popular power transistors widely adapted by the industry [13].

The MOSFETs were developed in the 1950's and matured in 1960's [14]. It enabled the first planar integrated circuit in 1959 [15]. The power MOSFETs saw its major growth in late 1960's to early 1970's evolving from VMOS to HEXFET. MOSFETS has become the workhorse for low to medium power by the power electronics industry since 1980 's. Its popularity was ushered in by the availability of avalanche-energy rated MOSFETs [16]. This is now extended to automotive applications for extreme ruggedness with low Rds(on) [17]. Figure 6 shows some common MOSFET packages widely used in industry.

For medium and high power applications, the insulatedgate bipolar transistor became the workhorse for industry in 1990's. Today, IGBTs capable of 1,000's of volts and 1,000 amperes are readily available from multiple vendors. For instance, Figure 7 shows the package of HiPak and Presspak $[18,19]$. The packages were developed to minimize parasitic inductance and capacitance for high reliability.

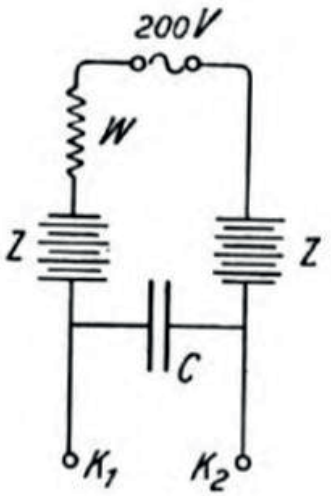

Greinacher, Ionometer

a)

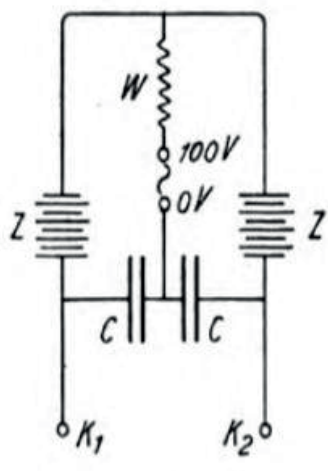

Physik. Zeitschr. XV, 1914

b)

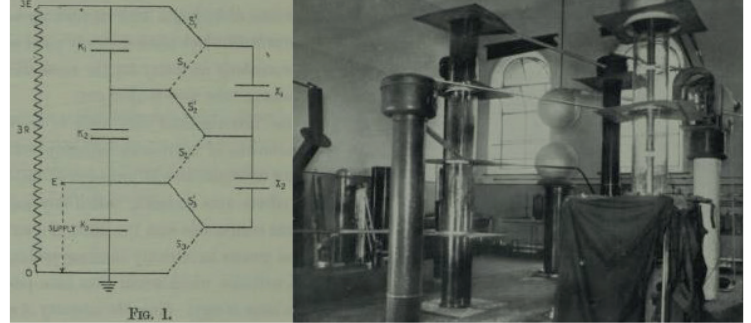

Fig. 3. The Cockcroft-Walton voltage multiplier (1932) - [8].

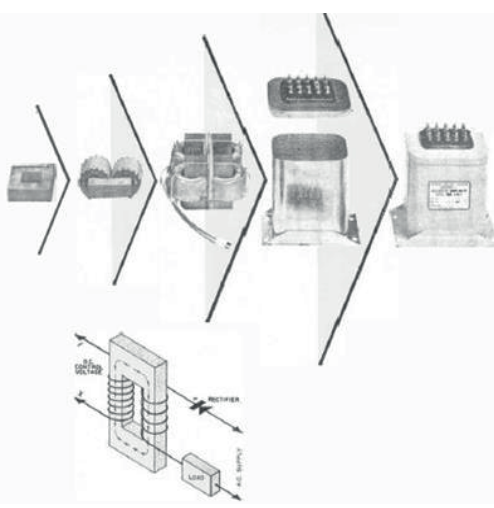

Fig. 4. The magnetic amplifier [9].

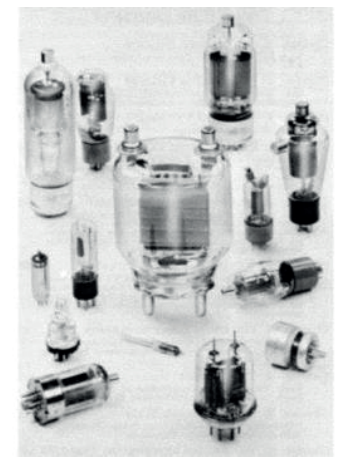

Fig. 5. The vacuum tube [10].
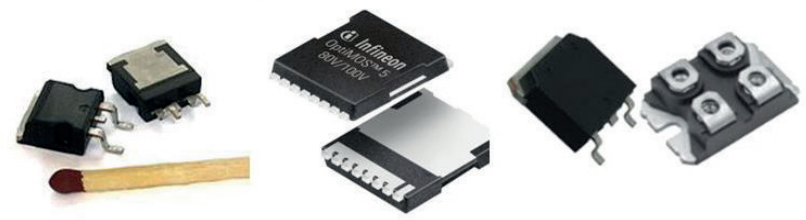

Fig. 6. MOSFETs for low- to medium-power applications.
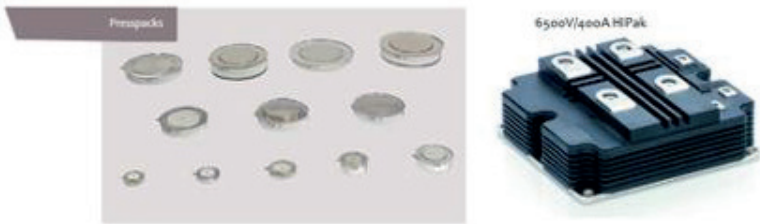

Fig. 7. MOSFETs for low- to medium-power applications.

Fig. 2. The Greinacher voltage multiplier (1914) - [7]. 


\section{RECENT ADVANCES}

A recent paper by the same author outlined emerging system applications and technological challenges in PEAS technology [1], proceeded by the original keynote presentation at APEC 2014. For instance, the six (6) system applications are recaptured here:

1. Renewable integration

2. Structure microgrids

3. All things grid connected

4. Transpiration electrification

5. Smart buildings/cities

6. Energy harvesting

The four (4) technological trends are recaptured:

1. Adiabatic power conversion

2. WBG devices

3. Power Supply on Chip (PwrSoC)

4. Multi-level conversion \& control

Much advancement has been made over the past five years, since the paper [1] was published in 2015. In the following, we will present a few of them to highlight the progress.

\section{A. WBG Devices Applications:}

Recently, wide-band-gap (WBG) devices are emerging, especially for special applications. Silicon-based Gallium Nitride $(\mathrm{GaN})$ and Silicon Carbide $(\mathrm{SiC})$ devices are the most noticeable and with most advanced products and many insertions to new designs. GaN devices are lateral highelectron-mobility transistors (HEMTs). For power switching applications, it enjoys low $\mathrm{Rds}(\mathrm{on})$ and low gate charge [20, 21]. Another significant advantage is its nature of free of reverse recovery charge (No pn-junctions). This allows cleaner totem-pole switching with lower current and voltage stresses. A third potential is its ability to be extended for bidirectional blocking capability.

$\mathrm{SiC}$ MOSEFETs are vertical devices that resemble $\mathrm{Si}$ MOSFETs. The reverse recovery charge is significantly smaller that of Si devices, allowing faster switching and lower voltage stress on switching devices. Devices in 10's of kilovolt are emerging on market to support the needs for high power designs [22, 23].

It is noted that $\mathrm{Si}$ power devices will continue play an important role because of its competitive or even better performances for low to medium power applications. However, both $\mathrm{GaN}$ and $\mathrm{SiC}$ has the potential to allow bidirectional voltage blocking capability, making them very appealing for future bidirectional power and energy processing applications [24, 25].

\section{B. Multilevel, Modular and Multi-timescale Technology:}

While a major technology breakthrough for power electronics is usually ushered in by the newer, faster and more powerful switching devices, as we discussed above, power electronic circuits play an important role in extending power switches' capability, in introducing controllability, and in improving reliability.

The modular multilevel converter (MMC) [26] is a quintessential example. As illustrated in Figure 8, the basic circuit of an MMC has two poles with bipolar supply voltages. Each pole can use a number of power modules connected in series to scale up the total voltage it can sustain. Each pole can also use a number of power modules connected in parallel to scale up current it can sustain. And hence the MMC can scale up power in order of magnitude higher than a single switch device is capable of. The multilevel approach brings improved reliability because of more even sharing of voltage and current [27]. This technological breakthrough enabled HVDC applications with voltage-source converters, replacing in many applications the traditional phase-controlled counterparts.

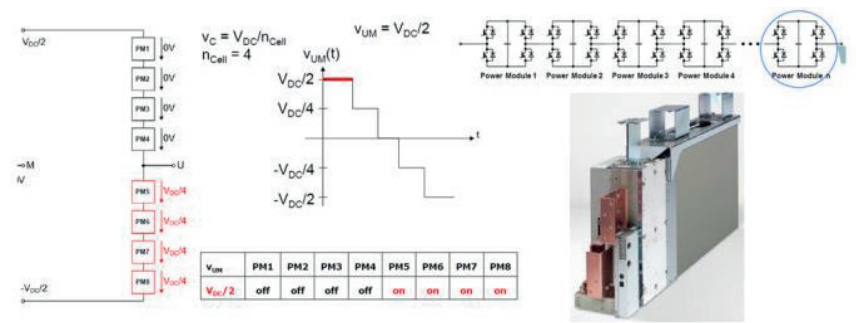

Fig. 8. modular multilevel converter represents a quintessential example where circuit techniques enhances the power switching capabilities.

\section{Megawatt System Design and Design Verifications:}

Another breakthrough happened in the area of megawatt systems design and design verification. The technology barrier for megawatt system design lies in two critical aspects of the design and design verification: 1) Megawatt systems are cost prohibitive for actual physical prototyping, unlike its counterparts for low power or medium power design, and 2) Simulation is too time consuming and is plagued with convergence problems to be useful for practical designs, not to mention for system deployment [28].

The first problem prevents engineering design teams to verify critical circuit behaviors beforehand. The entire system has to be then assembled on site without adequate prior simulation or testing to predict steady-state and, particularly, transient operations and performances. Consequently, problems frequently occur, particularly at interfaces where unintended interactions between modules and subsystems happen. Debugging potential hardware/software problems usually take long time, causing large schedule delays and unacceptable cost overruns. The second problem is often so severe that it renders the simulation tool virtually useless for design iteration and optimization, particularly at unit, subsystem and system levels [28].

The IEEE Power Electronics Magazine featured an article on a recent breakthrough in the Sep, 2020 issue [29]. The discrete-time, event-driven simulation technique has increased the speed by 3 orders of magnitude for complex systems and it is virtually free of convergence problems [2931]. Inside the algorithm is a revolutionary approach developed on the principal of event-driven simulation (As 
compared to traditional variable time steps). In addition, it employs a piece-wise linear model for the device characteristics. Figure 9 presents a case study of a $10 \mathrm{kV} 2-$ MW electronic energy router. The design has a total of 576 switching devices. As shown in the figure, the algorithm is 1,200 times faster than leading simulation software.

Figure 10 presents the simulated waveforms for a $50-\mathrm{kVA}$ solid-state transformer. The waveforms on the right are the zoomed-out view of the switch 1 voltage, current and inductor L1 current. The waveforms on the left are the zoomed-in view of the switch 1 voltage, current and inductor L1 current. Both sets of the waveforms were obtained within a single simulation run. It is clearly seen from the figure that the waveforms captured the transient behavior of the complex system with adequate details to predict voltage and current transient stresses on switches. This groundbreaking capability represents a breakthrough for megawatt system design and design verification. This breakthrough overcomes the two barriers we discussed above.

The simulation package can effectively support: 1) Very large converter system simulation, 2) Design optimization, 3) Sensitivity analysis and worst-case analysis, and 4) Device transient simulation. This breakthrough will usher in a new era in megawatt power electronic system design and verification and in other applications throughout the industry.

\section{Power Supply on Chip (PwrSoC) Technology:}

PwrSoC technology has made significant progress as it is evidenced by hundreds of papers published every year and dedicated single-topic workshop.

There are three major challenges in making a power supply on chip: 1) Silicon integration to include the controller, housekeeping and switching devices; 2) Fabrication of monolithic capacitors; and 3) Fabrication of monolithic inductors.

Success in silicon integration led to the establishment of the micro-power modules (Micro-modules for short) in the early 2000's [32]. As illustrated in the upper left corner of Figure 11, a micro-module integrates all active and discrete parts into a single package and then encapsulated into a chiplike final package. There are many products offering on the market as shown in Figure 11, for example, the LMT4608 can output $8 \mathrm{Adc}$ with greater than $85 \%$ typical efficiency in a package of $9 \times 15 \times 2.82 \mathrm{~mm} 3$ [32].

Fabrication of monolithic capacitors has made great progress. The achievable capacitance values are typically from 10's to 100's nF, approaching $500 \mathrm{nF} / \mathrm{mm} 2$ [33]. The so-called deep-trench capacitors are most producible and economical. Fabrications of monolithic inductors remain more challenging. The difficulty lies in the requirement for high permeability and low loss (High Q value). It is also less scalable [34]. The current achievable inductance values are typical in 10 's to $100 \mathrm{nH}$. Recent reported results are approaching $500 \mathrm{nH} / \mathrm{mm} 2$. They can also obtain high Q values on thin film-based techniques [35].

Because of the challenges outlined above, the Power Supply in Package (PSiP) technology is still prevalent in industry. This is the underlining reason as to why the micromodules are still popular. With the advancement of Si feature size reduction, the PSiP size is expected to continue to fall. There are many different approaches in implementing PSiP. For instance, Figure 12 shows one approach for integration of magnetic into a board $[36,37]$. This has demonstrated the capability of delivering 400A [36, 37]. Because of its proprietary nature, its applications have been limited.

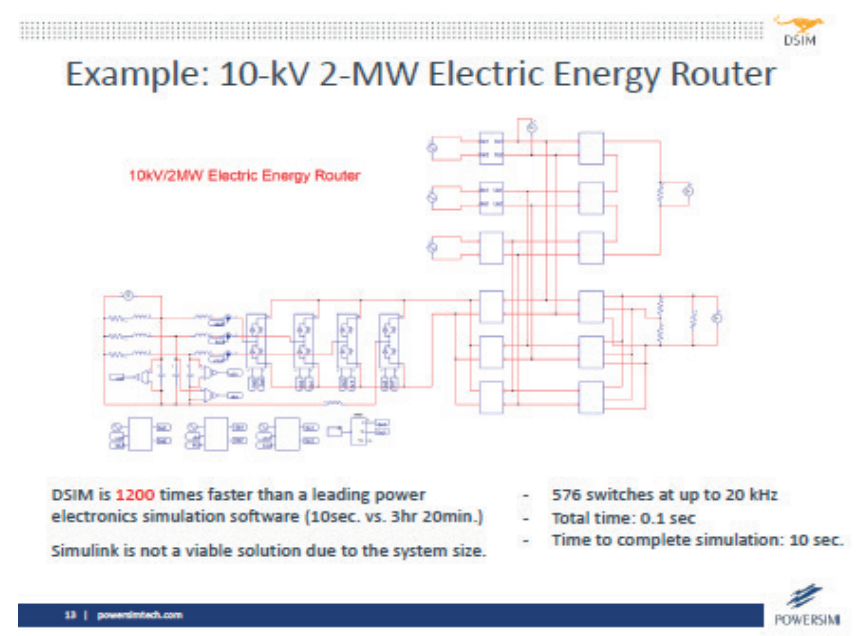

Fig. 9. case study 576 switches demonstrated breakthrough speed improvement and robustness
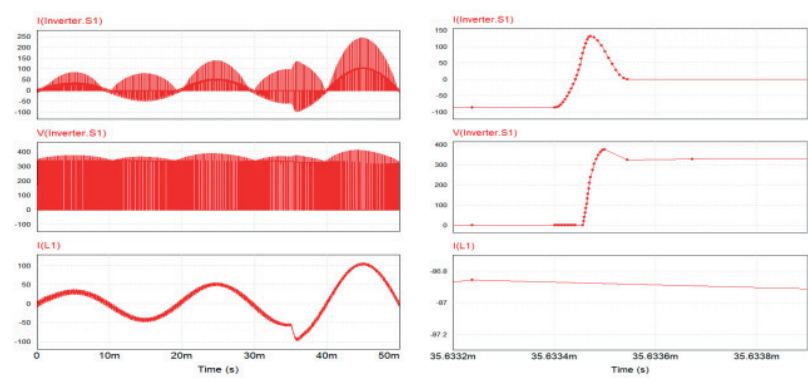

Fig. 10. Simulated waveforms for a 50kVA Energy Router: Left Inverter Switch 1 current, voltage and inductor L1 current waveforms (Zoomed out); Right - Inverter Switch 1 current, voltage and inductor L1 current waveforms (Zoomed in). Step-load change happens $35 \mathrm{~ms}$ as shown in the left figure.

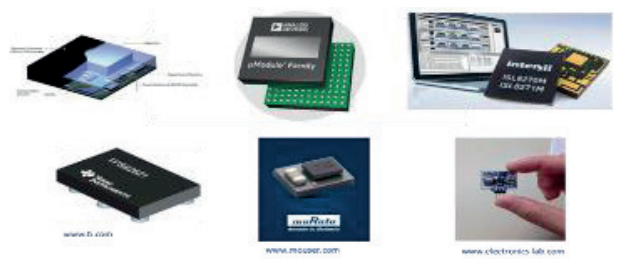

Fig. 11. Micro-power-module products offering

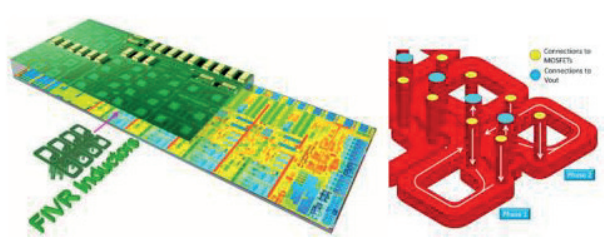

Fig. 12. Board integration of inductors (left and center) and switched-capacitor circuit (right) [36, 37]. 
For low power applications, modern version of the above mentioned switched-capacitor techniques can do an excellent job. Figure 13 shows a photo of a $3 \mathrm{~mW} 120 \mathrm{~V} / 230 \mathrm{~V}$ off line micro-power supply. The design employs the so-called charge pump circuit without any power inductors [38].

\section{CONTEMPORARY TRENDS}

The six (6) system application areas and four (4) technological trends described in [1] are still continuing today even after 5 years. The same author recently extended the discussion into the following top ten power electronics challenging areas $[39,40]$.

1. Powering smart buildings, smart factories, and smart infrastructure

2. Renewable energy integration and structured microgrids

3. EV drives and vehicle power systems

4. Ultra-fast and ultra-efficient chargers

5. Appliances : "White goods"

6. Server and data center power systems

7. Wireless power transfer

8. Powering IoT and wireless sensor networks

9. Storage and "power dump"

10. ATGC (All things grid connected), system integration, and dynamic control

In the following, we will provide a summary discussion. Interested readers can follow specific topic(s) through with the cited references.

\section{A. Powering Smart Buildings, Smart Factories, and Smart Infrastructure:}

Powering smart buildings, smart factories, or in general, smart infrastructure requires the PEAS technology to provide distributed smart systems. Distributed smart systems are electronic, digital, inter-connected and autonomous. All decisions are local and autonomously join the whole system [41]. Examples are smart homes, mart buildings, smart factories and smart infrastructure.

\section{B. Renewable Energy Integration and Structured Microgrids:}

Structured microgrids are natural vehicles for renewable energy integration into the power grids. Structured microgrids can provide the ultimate inertia for the grid's support. Detailed discussions can be found in $[1,6]$ on the structured microgrids (S $\mu \mathrm{Gs})$.

\section{EV Drives and Vehicle Power Systems:}

EV drive has made tremendous progress in recent years. The focus used to be on the performance and cost reduction. In recent years, the focus is increasingly on machine and drive integration to maximize performance and mileage per charge. With the advancement WBG devices, fully integrated machines and drive are emerging. A recent article [42] presents a good summary. Figure 14 shows s conceptual design of the $1 \mathrm{MW}$ integrated motor drive assembly for aircraft propulsion. The drive system is composed of six modular multilevel inverters mounted around the circumference of the stator. Each the module excites a dedicated set of three-phase windings. The project is currently in prototype development for completion in 2022.

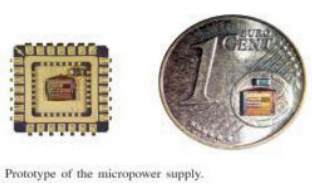

Fig. 13. A fully integrated power supply on chip for low-power offline applications [38].

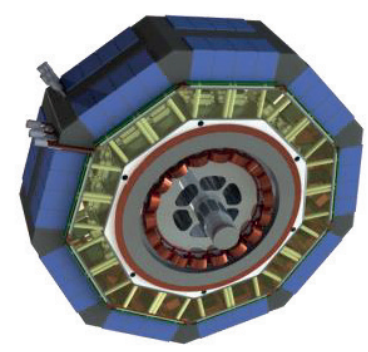

Fig. 14. A conceptual design of $1 \mathrm{MW}$ integrated motor drive assembly for aircraft propulsion (Courtesy of Jin Wang of Ohio State University).

\section{Ultra-fast and Ultra-efficient Chargers:}

Ultra-fast charging has emerged as key enabler for longdistance driving, for instance, from West coast to the East in the United States. The power required is nontrivial. For instance, an ultra-fast charging time of 10-15 minutes requires a total power of $350 \mathrm{~kW}$ for up to $80 \%$ of charge. If we use 4 ultra-fast chargers to form a charging station, analogous to a typical gas station, the power requirement is about 1.5 MW when factored inefficiency. For this amount of power, the best configuration is to use a structured microgrid to minimize its impact to grid power and to harvest available renewable energy in the local area $[6,43]$.

\section{E. Appliances: "White goods":}

PEAS technology is providing unparallel impetus for appliances industry to modernize its designs and products offering for more efficiency, light weight, and higher performance for the same or lower cost. An excellent detailed discussion on the topic can be found in [44].

\section{F. Servers and Data C'enter Power Systems:}

With the proliferation of the Internet, more and more data are stored and processed. Data storage and data center power processing has become an important issue for data-center operators. The electricity used for data center accounts for $2.2 \%$ of total electricity generated in 2015 and continues to 
grow [45]. The total ownership cost is composed of power, cooling, space and network. Once built, the power cost is dominant. The driving force behind this huge interest is simply economics: the cost electricity bill. For instance, a 10MW data center for 10 year life, the cost difference is $\$ 2.6 \mathrm{M}$ for electricity bills for a total efficiency of $93 \%$ and $94 \%$, assuming $\$ 0.10 \mathrm{~kW} / \mathrm{hr}$ [46].

So power electronic designer are driving for higher power efficiency from the utility interface to the rack and to the module and to the board. In recent years, multi-stage power processing regain momentum because it ability for squeeze last a few percent of efficiency out of the end-to-end efficiency. In this particularly regard, the near adiabatic power conversion technology helps to get the best efficiency [46] and the 99\% duty ratio technology can help improve the achievable power density, and hence further reducing the cost [46-49].

\section{G. Wireless Power Tranfer:}

Wireless power transfer is emerging as an important application area for power electronics. This is one typical example that technology (re)emergence spurred huge technological interest and progress that are enabled by new materials, devices, and techniques $[50,51]$. The IEEE Power Electronics Society (PELS) and the Microwave Technology and Techniques Society (MTTS) are jointly sponsored the Wireless Power Week (WPW) inaugurated in 2018 [52]. The WPW is being formalized as a joint venue for both PELS and MTTS to sponsor in order to provide an active international forum for people in this technological area to convene and to discuss their most recent progress and challenges.

One particularly interesting and potential groundbreaking area is the so-called the technology where Shannon meets Tesla $[53,54]$. This area is typically known as wireless information and power transfer (WIPT). The WIPT technology is promising to eliminate any dedicated power charging requirement. The power is transferred (beamed safely) together with the information content [54]. Here power electronics needs to be able to step up to provide efficient energy processing at hundreds of megahertz of the frequency spectrum safely.

\section{H. Powering IoT and Wireless Sensor Networks:}

Powering Internet $\mathrm{f}$ Things (IoTs) and wireless sensor networks is a closely related technological area to wireless power transfer. This is also coupled with wireless power transfer for implants and for various different forms of energy harvesting [55]. Ambient energy harvesting is one particularly interesting idea, but required efficient low-power devices and controllers to make it more feasible in real world applications [5], since the electromagnetic field in ambient is less $200 \mu \mathrm{W}[56]$.

\section{Storage and "Power Dump":}

Energy-storage has now come to speed up the transition from electric and electromechanical grids to electric, electromechanically and electronics grids (eGrids, for short).
The Australia Hornsdale battery system, supported by associated wind and solar farms, has proved it to be fast, accurate and valuable to the grid operators in supplying the ancillary services required for the South Austrian grid [57, 58] during peak demand.

Refer to Figure 15. The left graph is a time response of the frequency-control-ancillary-service (FCAS) response obtained from the traditional and expensive approach. Te black curve is the command and the red curve is the response. It can be seen that the response is sluggish and in many places the system was simply "stuck" for a while before responding the FCAS command. The right graph is the same FCAS response, but with the battery based power reserve. It is clearly seen that the command and response curves are virtually indistinguishable with one another. This unprecedented speed and accuracy has enabled the grid operator to stabilize the frequency while reduce the cost during the peak demand.

Economically, the Hornsdale facility has reduced the networks cost by $\$ 76 \mathrm{M}$ in 2019 over three major system outages, according to a report by Bloomberg. Recent reports indicated that major utilities are installing large battery facilities together with wind/solar farms to replacing aging gas-turbine-based peaker services with solar/battery peakers for improve accuracy, higher speed and lower cost [59].

\section{J. ATGC (All things grid connected), System Integration, and Dynamic Control:}

Another phenomenon with renewable energy is occasional excessive power because of the intermittent nature of renewables. There three typical solutions are: 1) Transmit it to other parts of the big grids; 2) More accurate forecasting of variations of renewable energy availability during the planning phase [60]; and 3) "Power dump" to a dummy load to absorb the excess such as combined heat and power [6].

All the above three options require the power grid to be actively controllable through PEAS technology. S $\mu$ Gs [1] and FeLPTs $[1,6]$ are the two major fundamental building blocks. A flexible electronic large power transformer (FeLPTs) is the combination of a traditional large power transformer and a flexible ac transmission (FACT) device $[61,62]$. Together with S $\mu$ Gs (with storage), a FeLPT provides traditional power processing for voltage scaling and galvanic isolation. It can also provide independent and active control to support the grid operations by providing ancillary services. Power substations equipped with these capabilities will be able to be flexible and autonomous, as first discussed in [63].

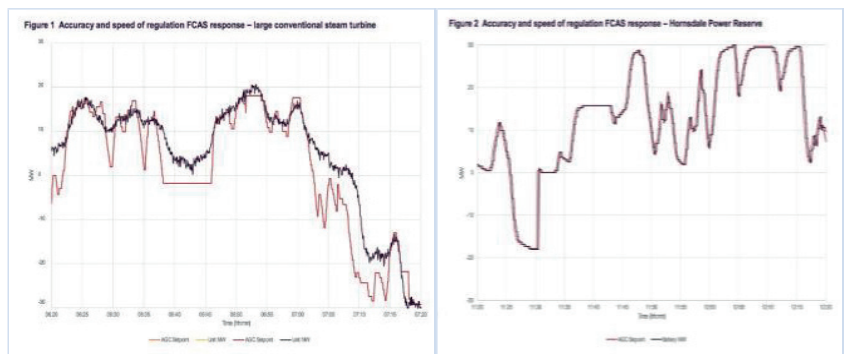

Fig. 15. Hornsdale battery installation has been proven as "Rapid, accurate and valuable." [58]. 
Flexible autonomous power substations (FAPS's) will serve as the basic functional building block for eGrids [6] to enable transactive energy, hence making the entire grid power flow controllable $[2-5,62]$. The benefits are many as discussed in [2-5]. A simple example is provided in Figure 16, where a weak feed can be strengthened by retrofitting existing power substations with FAPS's. The top portion is a voltage profile illustration and bottom indicates locations of substations $[63,64]$. This illustrates that the notion of "weak feeder or grid" may be a thing in the past once FAPS's are deployed widely. The initial cost for FAPS's can be easily offset by reduced cost for operation. As a matter of fact, the total ownership cost of FAPS's can be lower than traditional power substations because of high efficiency, renewable generation and ability for ancillary services [6].

\section{SUMMARY}

Power electronic technology can be traced back as early as 1903, when the mercury arc valve (rectifier) was invented. Power electronics technological progress is typically paced by the advances in switching devices, such as from thyratron to SCR to bipolar transistors to MOEFETS to IGBT, etc. But some of the key advances were ushered in by circuits and control techniques such as state-space averaging, current programming, multilevel power conversion, etc. Today, power electronics technology is advancing to power electronics and systems technology.

Emerging technology and system applications were presented previously. Recent advances are summarized in all emerging areas. WBG devices are entering mainstream for power processing. GaN will have an edge in low-power and high speed designs, while $\mathrm{SiC}$ will predominantly in high power applications. Si power devices will continue in lowpower and controller IC applications. Multilevel power conversion technique is continuing its progress from high power to medium and low power applications where complexity is traded for higher performance. A recent breakthrough in megawatt power system design verification allows simultaneous steady-state and transient behaviors to be simulated in a single simulation run for systems with hundreds or thousands of switching elements with record speed $(1,000 \mathrm{x}$ faster) and free of convergence problems. PwrSoC technology has progress with the capability of making monolithic capacitors up to $500 \mathrm{nF} / \mathrm{mm} 2$ and 500 $\mathrm{nH} / \mathrm{mm} 2$.

Contemporary trends are then summarized. The following areas represent the challenges and opportunities for PEAS technology: Powering smart buildings, smart factories, and smart infrastructure, Renewable energy integration and structured microgrids, EV drives and vehicle power systems, Ultra-fast and ultra-efficient chargers, Appliances - "White goods," Server and data center power systems' Wireless power transfer, Powering IoT and wireless sensor networks, Storage and "power dump," and ATGC (All things grid connected), system integration, and dynamic control. Each of the 10 areas represents significant challenges for PEAS technology. It is anticipated that an unprecedented growth of PEAS technology and the associated workforce will accompany the progress as we face the challenges and solve these problems.

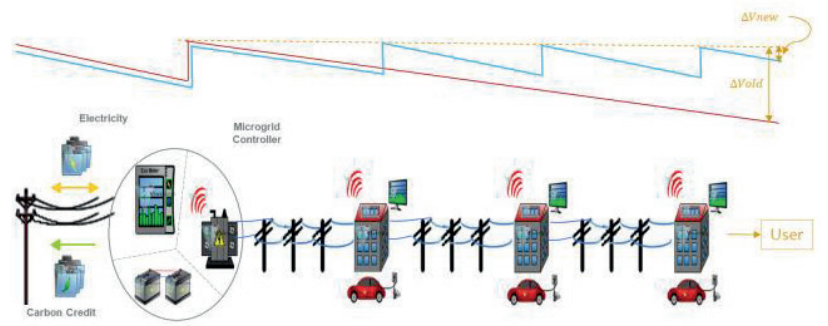

Fig. 16. Strengthening a weak feeder by deployment of $\mathrm{S} \mu \mathrm{Gs}$ and FeLPTs $[63,64]$

\section{REFERENCIAS}

[1] D. Tan, "Emerging System Applications and Technological Trends in Power Electronics," IEEE Power Electronics Magazine, vol. 2, no. 2, pp. 38-47, June 2015.

[2] D. Tan, "Electronictization: a foundation for grid modernization," CMP Journal of Electrical Engineering, Vol.1, No. 1, pp 1-10, Dec. 2015 (Invited Opening Paper for Inaugural Issue).

[3] D. Tan and D. Novosel, "Towards a (more) electronic grid," CES Transactions on Electric Machines and Systems, pp. 15-25, Mar 2017. (Invited Inaugural Issue Paper).

[4] D. Tan and D. Novosel, "Energy challenge, power electronics and systems (PEAS) technology and grid modernization," CPSS Transactions on Power Electronics and Applications, pp 3-11, Mar 2017 (Invited Inaugural Special Issue paper).

[5] D. Tan and D. Novosel, "Six (6) basic characteristics of the future grid," Journal of Grid Technologies, Southern Grid, Vo. 11, No. 10, pp. 2-9, Jan 2018 (Invited).

[6] D. Tan, "Structured microgrids (S $\mu \mathrm{Gs})$ and flexible electronic large transformers," CES TEMS, Dec, 2020 (Invited).

[7] Wikipedia, Mercury Arc Valve (Rectifier), Accessed on August 27, 2020 https://en.wikipedia.org/wiki/Mercuryarc_valve

[8] Wikipedia, Thyratron, Accessed on August 27, 2020 https://en.wikipedia.org/wiki/Thyratron

[9] Greinacher, H., "Das Ionometer und seine Verwendung zur Messung von Radium- und Röntgenstrahlen" (The ionometer and its application to the measurement of radium- and Röntgen rays) Physikalische Zeitschrift (in German), 15, pp. 410-415, 1914.

[10] J. D. Cockcroft and E. T. Walton, "Production of highvelocity positive ions," Proceedings of the Loyal Society, A, vol. 129, pp. 619-630, 1932.

[11] K. Gilmore, "Magnetic amplifiers: how they work and what they do," Popular Electronics, pp. 71-75 and p. 109, July 1960.

[12] R. B. Tomer, "Getting most out of your vacuum tubes," Photofact Pubs, 1960. 
[13] John G. Kassakian, "Power Electronics: Past, Present and Future," APEC2020.

[14] C.-T. Sah, "Evolution of the MOS transistor - from conception to VLSI," IEEE Proceedings, pp5 12801326, Oct 1988.

[15] J. A. Hoerni and R. N. Noyce, "Semiconductor planar process and integrated circuit, 1959" IEEE Milestones in Electrical Engineering and Computing, Palo Alto, CA (Dedicated on May 8, 2009).

[16] R.R. Stoltenburg, "Boundary of Power MOSFET, unclamped inductive switching (UIS), avalanche current capability," IEEE APEC Proceedings, pp. 359364, 1989

[17] A. Murray, H. Davis, J. Cao, K. Spring, and T. McDonald, "New power MOSFET technology with extreme ruggedness and ultra-low Rds(on) to Q101 for automotive applications," PCIM Proceedings, Power Conversion paper 4.5, Nurnbergpp. 103-107, 2000.

[18] R. Schnell et al, "Next-generation high voltage packaging and IBGT/diode technology," PCIM Asia, 2016.

[19] M. Schulz, "Heavy Duty Applications - A Challenge for Power Semiconductor Devices," PCIM Asia, 2016.

[20] T. P. Chow, "Wide bandgap semiconductor power devices for energy efficient systems," 2nd IEEE Workshop on WBG Power Devices and Applications (WiPDA), pp. 402-405, 2015.

[21] D. Tan, "WBG devices for space applications," 1st IEEE Workshop on WBG Power Devices and Applications (WiPDA), 2014 (Opening Keynote for the Series).

[22] G. Deboy, M. Tery, O. Haeberlen, and D. Neumayi, "Si, SiC, and $\mathrm{GaN}$ power devices, an unbiased view on key performance indicators," IEEE IEDM, pp. 20212024, 2016.

[23] B. Passmore et al., "The next generation of high voltage $(10 \mathrm{kV})$ silicon carbide power modules," 4th IEEE Workshop on Wide Bandgap Power Devices and Applications (WiPDA), pp. 1-4, 2016.

[24] T. Morita, et al, "650V $3.1 \mathrm{mOhm} * \mathrm{~cm} 2 \mathrm{GaN}$ based monolithic bidirectional switch using normally-off gate injection transistor," IEEE IEDM, Washington DC, pp. 865-868, 2007.

[25] S. Chowdhury, C. W. Hitchcock, Z. Stum, R. P. Dahal, I. B. Bhat and T. P. Chow, "Operating Principles, Design Considerations, and Experimental Characteristics of High-Voltage 4H-SiC Bidirectional IGBTs," IEEE Transactions on Electron Devices, vol. 64, no. 3, pp. 888-896, March 2017.

[26] R. Marquardt, "Modular Multilevel Converter: An universal concept for HVDC-Networks and extended DC-Bus-applications," The 2010 International Power Electronics Conference - ECCE ASIA -, Sapporo, pp. 502-507, 2010.

[27] D. Retzmann, "Modular multilevel converters: Technology and Principles," Siemens Energy Sector, Siemens AG, 2009.

[28] D. Tan, "Power Electronics in 2025 and Beyond: A Focus on Power Electronics and Systems Technology,"
IEEE Power Electronics Magazine, vol. 4, no. 4, pp. 33-36, Dec. 2017.

[29] Z. M. Zhao, D. Tan, et al, "A breakthrough in megawatt power system design," IEEE Power Electronics Magazine, pp. 36-43, Sep 2020.

[30] Z. Zhao, D. Tan and K. Li, "Transient Behaviors of Multiscale Megawatt Power Electronics Systems - Part I: Characteristics and Analysis, " IEEE Journal of Emerging and Selected Topics in Power Electronics, vol. 7, no. 1, pp. 7-17, March 2019.

[31] Z. Zhao, D. Tan, K. Li and L. Yuan, "Transient Behaviors of Multiscale Megawatt Power Electronics Systems - Part II: Design Techniques and Practical Applications," IEEE Journal of Emerging and Selected Topics in Power Electronics, vol. 7, no. 1, pp. 18-29, March 2019.

[32] Linear Technology (Analog Devices, now), LTM4608 Datasheet, 2007.

[33] F. Nodet, "Low-profile \& multi-terminal Silicon capacitors," IEEE Workshop on Power Supply on Chip (PwrSoC), 2018, Talk 3.3 (Murata/ipdia).

[34] C. R. Sullivan, et al, "On size and magnetics: Why small efficient power inductor are rare," Symposium on 3D Power Electronics Integration and Manufacturing (3D-PEIM, pp. 1-4), 2016.

[35] N. Sturchen, Integrated power management with ferromagnetic thin-film power inductors," IEEE Workshop on PwrSoC, 2018, Talk 4.2.

[36] E. A. Burton et al., "FIVR - Fully integrated voltage regulators on 4th generation Intel ${ }^{\circledR}$ Core ${ }^{\mathrm{TM}}$ SoCs," IEEE Applied Power Electronics Conference and Exposition, TX, pp. 432-439, 2014.

[37] P. R. Morrow, J. Ted DiBene et al, "Design and fabrication of on-chip coupled inductors integrated with magnetic material for voltage regulators," IEEE Transactions on Magnetics, pp. 1678-1686, June 2011.

[38] D. Lutz, P. Renz and B. Wicht, "An Integrated 3-mW 120/230-V AC Mains Micropower Supply," IEEE Journal of Emerging and Selected Topics in Power Electronics, vol. 6, no. 2, pp. 581-591, June 2018.

[39] D. Tan, "Power electronics and top challenges," CES Cloud Forum: Leaders in Electrotechnology, Inaugural Talk, Aug. 2020

[40] D. Tan, "Power electronics challenges," Personal communications with M. Tse, et al, Apr 2018.

[41] D. Tan, "Powering smart factories," CATS Panel on Smart Manufacturing, May, 2018 (Invited Keynote).

[42] T. M. Jahns and B. Sarlioglu, "The quest for the disappearing motor drive," IEEE Power Electronic Magazine, pp. 18-27, Sep 2020.

[43] D. Tan, "Challenges in fast and ultra-fast EV chargers," 2nd IEEE ITES Asia-Pacific, Aug 2017 (Keynote).

[44] J. Lai, "Power electronics for household appliance revolution," IEEE ECCE-Asia, 2015 (Keynote).

[45] O. Lior, "Data center facility basic," Challenges in Modern Data Centers Management, 2015.

[46] D. Tan, "Power-conversion technology is going adiabatic," IEEE Power Electronics Magazine, pp. 4753, Dec 2015. 
[47] D. Tan, et al, "A review of intermediate bus architecture: a system perspective," IEEE Journal of Emerging and Selected Topics in Power Electronics, vol.2, no. 3, pp. 363-372, Sep 2014.

[48] D. Tan, "Multi-stage power processing - Near adiabatic conversion and high power density," IEEE Power Electronics Applications Conference, 2018 (Keynote).

[49] D. Tan, "Ultra high power density demands 99\% efficiency and 99\% duty ratio," IEEE ECCE Plenary Keynote, Oct 2019.

[50] Wikipedia, Wireless power transfer, accessed on August 21, 2020

https://en.wikipedia.org/wiki/Wireless_power_transfer

[51] D. Tan, "Power electronics, transportation electrification, distributed smart systems, and beyond," Inaugural IEEE PELS Wireless Power workshop (WoW), Jun 2015 (Opening keynote of the inaugural workshop).

[52] D. Tan, "Powering distributed smart systems," Inaugural IEEE Wireless Power Week, Montreal, 2018 (Keynote).

[53] P. Grover and A. Sahai, "Shannon meets Tesla: Wireless information and power transfer," 2010 IEEE International Symposium on Information Theory, Austin, TX, pp. 2363-2367, 2010.

[54] T. D. P. Perera, D. N. K. Jayakody, S. Chatzinotas and V. Sharma, "Wireless Information and Power Transfer: Issues, Advances, and Challenges," 2017 IEEE 86th Vehicular Technology Conference (VTC-Fall), Toronto, ON, 2017.

[55] J. A. Paradiso and T. Starner, "Energy harvesting for mobile and wireless electronics," Pervasive Computing Magazine, published by IEEE CS and IEEE ComSoc, pp. 18-27, Jan-Mar 2005.

[56] H. Zhang, Y-X Guo, et al, "Cooperative integration of RF energy harvesting and dedicated WPT for wireless sensor networks," IEEE Microwave and Wireless Components Letters, Mar 2019.

[57] D. Tan, "Batteries: the ultimate inertia for renewable integration," IEEE T\&D Show, April 2018 (Invited Super Session Panelist).

[58] "Initial operation of the Hornsdale power reserve battery energy storage system," Australian Energy Market Operator (AEMO), April 5, 2018.

[59] Energy Storage News, "8minutenergy: We can do solar peaker plants at the half the price of gas". https://www.energy-storage.news/news/8minutenergywe-can-do-solar-peaker-plants-at-half-the-cost-of-gas
[60] "IBM announces cloud-based enterprise-wide analytics for energy companies," Winder Power Engineering \& Development, Feb 2015.

[61] B. Fardanesh, B. Shperling, E. Uzunovic and S. Zelingher, "Multi-converter FACTS devices: the generalized unified power flow controller (GUPFC)," 2000 Power Engineering Society Summer Meeting, pp. 1020-1025, 2000.

[62] Z. Huang, F. F. Wu, et al, "Application of UPFC in interconnected power systems - Modeling, interface, control strategy and case study," IEEE Transactions on Power Systems, vol. 15, no. 2, , pp. 817-824 May 2000.

[63] D. Tan, "Batteries, Grids, and flexible autonomous power substation," IEEE eGRD Workshop, 2019 (Keynote) (Available through IEEE PELS and ES member resource center).

[64] C. Schweagerl, "Technical, economic and environmental benefits of microgrids operation," Siemens, Jan 2010.

\section{BIOGRAPHIES}

Dr. Don Tan, is a Distinguished Engineer/Fellow/Chief Engineer for Power Products with Northrop Grumman Space Systems. He earned his $\mathrm{PhD}$ from Caltech and is an IEEE fellow. Well-recognized as an authority in ultra-efficient power conversion and energy systems, he has pioneered breakthrough innovations with high-impact industry firsts and record performances that "significantly enhance national security." Don has delivered 50+ keynotes and invited presentations across the globe. He has been serving Chair/Vice Chair, IEEE Next-Gen Financial System by the Board of Directors since 2018. He is Chair of IEEE Field Award on Transportation Electrification Technologies since 2019. He was Director, IEEE Board of Directors, 2017-2018. $\mathrm{He}$ is Steering Committee Chair for IEEE eGrid Workshop jointly sponsored by Power Electronics Society (PELS) and Power and Energy Society (PES). He has served on many IEEE PELS in many capacities, including Long Range Planning Committee Chair, Nomination Committee Chair, Society President, Editor-in-Chief (Founding) for IEEE Journal of Emerging and Selected Topics in Power Electronics, Vice President for Operations, Guest Editor-inChief for IEEE Transactions on Power Electronics and IEEE Transactions on Industry Applications, Fellow Committee, Vice President for Meetings, and Associated Editor for IEEE Transactions on Power Electronics. He has served and is serving on many major national and international award/review/selection committees. 